\title{
PROGRAMA DE FORMACIÓN DOCENTE EN FACULTAD DE QUÍMICA
}

\author{
MÉNDEZ, Shirley \\ RODRÍGUEZ AYÁN, María Noel \\ AMAYA, Alejandro
}

\section{INTRODUCCIÓN}

La formación docente de los profesores universitarios es fundamental cuando se habla de calidad universitaria. A nivel internacional se observa que esta formación es adecuada en lo referido a los contenidos de las distintas asignaturas y que está casi siempre asegurada. Sin embargo la formación en pedagogía y en didáctica no está tan clara. Estas áreas son de mucha importancia debido a la influencia que tiene la calidad de los docentes en los resultados de los estudiantes (OCDE, 2005).El requerimiento de una formación continua para el profesorado aparece en distintos informes (ATTALI, 1997; BRICALL, 2000; DEARING 1997) y en la mayoría de los casos la formación docente no está prevista como obligatoria.

\footnotetext{
El profesorado cuenta. Cuenta para influir en el aprendizaje de los alumnos. Cuenta para mejorar la calidad de la educación que las escuelas e institutos llevan a cabo día a día. Cuenta en definitiva como una profesión necesaria e

imprescindible para la sociedad del conocimiento." (MARCELO, 2007, P. 66)
}

Por ello la profesionalización es un proceso continuo que se construye a partir de integrar la identidad profesional, las competencias profesionales, los requisitos de acceso, la formación asociada, el desarrollo de la carrera profesional y la evaluación del desempeño (TEJADA, 2011).Este proceso de formación incluye la reflexión sobre el ejercicio profesional, las investigaciones de aula incorporándolas a la práctica, evaluación de la eficacia de las estrategias de enseñanza utilizadas. También es importante conocer la necesidad de formación en distintas áreas, el trabajo interdisciplinario y la incorporación de las tecnologías de la información y la comunicación (MAS Y TEJADA, 2012) 
Desde el momento de su creación la Universidad de la República plantea como sus funciones principales enseñanza, investigación y extensión estando las tres a cargo de los docentes. A partir de los años ochenta se ha visto un incremento de la matrícula universitaria lo que ha representado un aumento en la heterogeneidad de los estudiantes y también de los docentes. En este orden la Universidad de la República muestra una clara preocupación por la formación docente. Existen programas de formación docente centrales, transversales a toda la Universidad, desde 2001. En el marco de dichos programas la Comisión Sectorial de Enseñanza de la Universidad organiza actividades dirigidas a todos los docentes universitarios, tales como talleres y conferencias, procurando abordar temáticas de interés general, trayendo muchas veces conferencistas extranjeros como invitados. Este programa central tiene su correlato particular en cada Facultad, puesto que la didáctica debe abordarse en un determinado marco de contenidos, lo que la torna una disciplina específica y no genérica.

La Facultad de Química en particular tiene una larga y vasta trayectoria dentro de la Universidad de la República como institución productora de conocimientos científicos, teniendo casi todos sus docentes el grado de Doctor. Sin embargo, la institución es consciente de que aún hace falta que los docentes además se capaciten en áreas didáctico-pedagógicas para el mejor ejercicio de su labor de enseñanza. La Unidad Académica de Educación Química (Unadeq) es la estructura académica que tiene dentro de sus funciones la responsabilidad de implementar acciones para promover la formación de los docentes de la Facultad de Química a través de su Programa de Formación Docente.

\section{METODOLOGÍA}

Desde su creación en 1998 la Unadeq viene realizando distintas acciones respecto a la formación de los docentes de la Facultad de Química. Inicialmente se realizaron talleres para docentes, fundamentalmente dirigidos a explorar las necesidades del cuerpo docente en relación a su formación didáctico-pedagógica. Así se observó, por ejemplo, que en general el cuerpo docente de la Facultad prefiere una formación esencialmente práctica, que le 
permita cumplir eficazmente con la función de enseñanza, expresando cierta reticencia al abordaje de la formación docente desde la teoría pedagógica. Interesa señalar que los docentes de la Facultad de Química en general están alineados con la concepción de que la investigación permite mejorar la enseñanza. Este concepto fue propuesto hace más de 30 años por Hoffmann (1984), junto a la concepción de que también la enseñanza permite mejorar la investigación, porque en realidad enseñanza e investigación no deben ser tratadas como actividades disociadas: propone que pensemos en términos de distintas audiencias a quien el docente-investigador debe dirigirse.

Con el tiempo desde la Unadeq se comenzaron a implementar cursos, no solo dirigidos a docentes de la Facultad de Química sino también abierto a otras Facultades de la Udelar, así como a docentes de Enseñanza Secundaria. Las temáticas de los cursos fueron surgiendo de relevamientos realizados, primero de manera informal, y posteriormente de manera más sistemática, mediante encuestas a los docentes y entrevistas semiestructuradas. Entre los cursos inicialmente dictados se destacan Planificación Educativa, Mediciones cuali-cuantitativas y Tendencias didácticas en la enseñanza de las Ciencias Naturales. Estos dos últimos determinaron la publicación de dos libros: Mediciones cuali-cuantitativas (RODRÍGUEZ AYÁN Y SOUBIRÓN, 2005) y Algunas tendencias didácticas en la enseñanza de las Ciencias Naturales y Exactas (FIORE, HERMIDA, LEYMONIÉ, MíGUEZ, RODRÍGUEZ AYÁN Y SOUBIRÓN, 2006). Cabe señalar que el curso de Tendencias didácticas fue un esfuerzo coordinado desde la Facultad de Química con participación de las Facultades de Ingeniería y de Ciencias, con coparticipación de los docentes de las tres Unidades de Enseñanza.

A partir del año 2015 se establecen tres líneas de trabajo relacionadas con la formación de los docentes universitarios:

i. Formación docente a través de cursos creditizables.

ii. Ciclos de Conferencias sobre distintos temas vinculados a la práctica docente.

iii. Evaluación docente integral, entendiendo por tal la evaluación resultante de la aplicación de diferentes técnicas de recogida de datos: autoevaluación docente, evaluación por pares, evaluación estudiantil, evaluación por parte del superior jerárquico y observación no participante de 
clases. Se debe señalar que no siempre se ha podido desarrollar la evaluación haciendo uso de todas las fuentes de datos mencionadas.

En base a estas líneas, durante el año 2015 se desarrollaron las siguientes actividades:

i. En la formación docentes en base a cursos se realizaron los siguientes: Tópicos de Psicología Educación: Módulo Motivación y Diseño y Análisis de Pruebas de Múltiple Opción. Este último curso ha sido dictado sistemáticamente desde 2013, con gran aceptación no solo por parte de docentes de la Facultad de Química sino también de otras Facultades. Actualmente se está trabajando en la edición de un libro de texto para el curso, similarmente a lo realizado para los cursos Planificación educativa y Algunas tendencias didácticas en la enseñanza de las ciencias naturales y exactas.

ii. Los ciclos de conferencias se realizaron uno en cada semestre de manera que en el primero se realizaron cuatro presentaciones que abarcaron los siguientes temas:

- Innovación en el aula: Matemática en Facultad de Arquitectura,2 años de intentos y algunos logros.

- Educación abierta

- Tecnología multimedia

- ¿Cómo aprenden los estudiantes universitarios?

En el segundo semestre los temas tratados fueron:

- Gestión del estrés en la evaluación

- Calidad en educación superior.

En relación a este último tema se realizó como cierre del ciclo una mesa redonda donde se invitó a participar no solo a docentes sino también a integrantes del Parlamento uruguayo, para debatir sobre calidad en educación superior en contextos de masividad.

iii. La evaluación docente se efectúa dando prioridad a los aspectos formativos. El abordaje se realiza tanto desde el punto de vista de las opiniones de los estudiantes como de superiores, de sus pares y de especialistas en aspectos didácticos. Los docentes que solicitan ser evaluados por los estudiantes valoran mucho esta instancia ya que la información que reciben les permite revisar su práctica docente y evaluar las estrategias utilizadas en el aula o en el laboratorio. En el año 2015 fueron evaluados docentes de 
diferentes asignaturas tanto teóricas como de laboratorio, así como de diferente trayectoria docente. A solicitud de la responsable del área microbiológica se hizo la evaluación completa de un curso de Microbiología la que consistió en:

- Evaluación por parte de los estudiantes asistentes a las clases presenciales.

- Evaluación por parte de pares docentes del mismo curso.

- Evaluación por observación de clases por parte de la Unadeq.

- Autoevaluación.

Con respecto al presente año ya se consideran establecidas estas tres líneas de trabajo y se están llevando a cabo de la siguiente manera:

En la línea de cursos se dictarán dentro de Tópicos en Psicología de la Educación dos módulos, uno correspondiente a Motivación, ya dictado en 2015, y un nuevo curso sobre Estilos y Enfoques de Aprendizaje.

Se está realizando un ciclo de siete conferencias vinculadas con las investigaciones en Neurociencias y el aprendizaje. Abarcan temas referentes a:

- Organización del Sistema Nervioso Central

- Neurociencias y educación

- Neurobiología del sueño

- Emociones y stress

- Neuroadaptaciones en la adicción de drogas de abuso

- Neurobiología de la motivación.

- Cognición y matemática

Con respecto a la evaluación docente se realiza este año evaluación docente en base a la opinión estudiantil.

\section{RESULTADOS Y DISCUSIÓN}

Las tres líneas de formación docente buscan atender las demandas de los docentes de la Facultad de Química desde distintos niveles de involucramiento. Considerando que las actividades se realizan durante el año lectivo, y en la misma los docentes están con sus actividades de enseñanza, investigación y extensión, el hecho de tener dos opciones (presencial y virtual, formal y no formal) les permite decidir en base al tiempo disponible para dedicarle a la actividad y también en relación con sus sus intereses. Por eso 
desde la Unadeq se ha tenido en cuenta a aquellos docentes que desean una formación más intensa, formal (obtención de créditos) y que la misma pueda incorporarse al plan de estudios de posgrado y a aquellos otros que desean estar al día con las nuevas investigaciones en educación pero no disponen del tiempo para realizar cursos extensos.

Los cursos son virtuales en su totalidad y tienen una carga horaria de 60 horas de dedicación por parte de los participantes. En ellos se busca la participación en los foros, a través de discusiones grupales o de reflexiones críticas de temas específicos. La evaluación final se realiza a través de la elaboración de un trabajo personal de análisis de los temas tratados incorporando la reflexión personal del participante. En el caso de los cursos dictados en el año 2015 la asistencia se dividió en $27 \%$ de docentes de la Facultad de Química, 14\% de docentes de otros servicios de la Universidad de la República y $59 \%$ de docentes de otros subsistemas educativos uruguayos, principalmente Enseñanza Secundaria y Enseñanza Técnico Profesional (de carácter terciario).

En cuanto a las conferencias, las mismas son presenciales y se establece un día y un horario fijo en cada semestre para que los docentes interesados puedan organizar sus actividades para asistir a las mismas. En estas se pudo observar el interés de los docentes por la práctica, por la búsqueda de estrategias de enseñanza, de conocer las características de los estudiantes en este siglo XXI ya avanzado, en las posibilidades que nos brindan las redes y la educación no presencial. Dentro de estos temas la conferencia que tuvo mayor participación fue la referida a " ¿Cómo aprenden los estudiantes universitarios?" donde se profundizó en los enfoques de aprendizaje de los estudiantes universitarios y cómo desde la docencia podemos fortalecerlo. La concurrencia a las conferencias fue variada y los docentes mostraron interés en este modo de formación así como sugirieron ciertos temas en los que les interesaría tener más información. Con respecto a la asistencia a los Ciclos de Formación Docente 2015 la concurrencia de docentes de Facultad de Química fue de un 65\% de los asistentes mientras que docentes de otros servicios de la Universidad de la República fue de un $19 \%$ y de otros servicios ajenos a dicha universidad fue de un $16 \%$. 
Cabe destacar que participaron docentes de los distintos grados en el escalafón docente: grado 1 (Ayudante), grado 2 (Asistente), grado 3 (Profesor Adjunto), grado 4 (Profesor Agregado) y grado 5 (Profesor Catedrático). Ello constituye una muestra de que la preocupación por la formación es transversal a la carrera docente.

En cuanto a la evaluación docente, esta es solicitada por más de 20 docentes al año, siendo el instrumento más comúnmente empleado las encuestas de opinión estudiantil. En algunos casos la evaluación no fue solicitada por los docentes sino por encargados de cursos e incluyen también opiniones de pares, observaciones metodológicas y opiniones de referentes académicos del curso dictado.

\section{CONCLUSIONES}

Los docentes de la Facultad de Química participan de las actividades de formación docente que organiza la Unadeq. El hecho de encontrarnos cada día con un cuerpo estudiantil heterogéneo, con trayectos educativos previos variados y metas diferentes hace que lo docentes debamos estar en un continuo proceso de formación que no solo se base en brindar información sino también promueva la reflexión y el análisis de las prácticas personales y del conjunto de los docentes. Al decir de Bain $(2005,193)$ considerar que la enseñanza "solo tiene lugar cuando hay aprendizaje" entonces es fundamental el establecer entornos que propicien estos aprendizajes. Eso es lo que a través de las distintas actividades mencionadas se busca lograr estableciendo sobre todo un puente de dialogo para poder saber cuáles son las inquietudes y las necesidades de los docentes de este Servicio.

\section{BIBLIOGRAFÍA}

BAIN, K (2005) Lo que hacen los mejores profesores de la universidad. Valencia, Publicaciones de la Universidad de Valencia. 
FIORE, E.; HERMIDA, A.; LEYMONIÉ, J.; MÍGUEZ, M.; RODRÍGUEZ AYÁN, M.N.; SOUBIRÓN, E. Algunas tendencias didácticas en la enseñanza de las Ciencias Naturales y Exactas. Montevideo: Zonalibro, 2006.

HOFFMANN, R. Research Strategy: Teach. American Scientist, 1984, 84, p. 20-22.

MARCELO, C. (2007). La formación docente en la sociedad del conocimiento y la información: avances y temas pendientes. Olhar de professor, Ponta Grossa, 10(1): 63-90, 2007. Disponíble en <http://www.uepg.br/olhardeprofessor >

MAS, O; TEJADA, J. (2013). Funciones y competencias en la docencia universitaria. Madrid: Síntesis.

OCDE (2009). Los docentes son importantes: atraer, formar y conservar a los docentes eficientes. París: OCDE.

RODRÍGUEZ AYÁN, M.N.; SOUBIRÓN, E. Mediciones cuali-cuantitativas. Montevideo: Zonalibro, 2006.

TEJADA, J (2011). Evaluación del desarrollo profesional docente basado en competencias. En MARCELO, C (coord.) Evaluación del desarrollo profesional docente. Barcelona, Editorial Da Vinci. 